RESEARCH ARTICLE

\title{
Nasal Carriage Frequency of Staphylococcus aureus According to Education Years of Medical Students*
}

\author{
Oğuz Karabay¹, Zeynep Unus Yılmaz², Melike Kuvvet², Yusuf Taşpınar², Emel Akce², \\ Ertuğrul Güçlü', Aziz Öğütlü \\ ${ }^{1}$ Department of Infectious Diseases and Clinical Microbiology, Health Sciences Institute, Sakarya, Turkey \\ ${ }^{2}$ Sakarya University Faculty of Medicine, Sakarya, Turkey
}

\begin{abstract}
Objective: Nasal carriage of Staphylococcus aureus (NCSa) is one of the most important risk factors for nosocomial infection. Although there are numerous studies concerning NCSa frequency among medical staff, studies dealing specifically with medical students are less common. We aimed to investigate NCSa frequency among medical students and to compare the rates of clinical students (CS), comprising interns and stagers, with those of preclinical students (PS).
\end{abstract}

Materials and Methods: This study was performed at Sakarya University Medical School. All students were invited to participate into the study. Nasal swab samples were taken anterior nares from 146 medical students $(P S=82, C S=64)$. The samples were inoculated into the Chapman agar medium, and incubated at $37^{\circ} \mathrm{C}$ for 48 hours. Antibiotic sensitivity tests were performed on these colonies. The obtained data were analyzed statistically. Chi-square test was used for qualitative variables and $t$ test was used as quantitative data. $\mathrm{P}<0.05$ was considered significant.

Results: A total of 146 students were agreed to participate in the study (53 subjects were male and 93 were female). While NCSa frequency within the PS group was $2 / 82(2.4 \%)$, that within the CS group was 7/64 (10.9\%) ( $p=0.042)$. Methicillin resistance was not detected in any subject.

Conclusion: As a result, the frequency of NCSa in the CS group (10.9\%) was found to be about 4 times higher than that in the PS group (2.4\%). We recommend that clinical students should be expected to observe infection-control precautions. J Microbiol Infect Dis 2016; 6(3): 103-106

Keywords: Nasal carriage, Staphylococcus aureus, medical student

\section{INTRODUCTION}

Staphylococcus aureus is a coagulase-positive, catalase-positive, and gram-positive bacterium that tends to form in clumps. S. aureus can cause many forms of both mild and serious infections, such as superficial skin lesions, osteomyelitis, endocarditis, infection of surgical wounds, food poisoning, toxic shock syndrome, and pneumonia [1]. S. aureus can easily colonize various parts of the body such as the axillae, the perineum and especially the one-third proximal aspect of the nose. Accordingly, the term "nasal carriage of S. aureus" (NCSa) is used. Exposure to NCSa is one of the most important risk factors for for staphylococcal infection [2]. There are three different patterns of NCSa occurrence within the general population:

1. Persistent carriage, affecting about $20 \%$ of the population, occurring more commonly in children than in adults, and consisting of a single strain; [3]

Correspondence: Oğuz Karabay, Sakarya University, Department of Infectious Diseases and Clinical Microbiology, Health Sciences Institute, Sakarya, Turkey Email: okarabay@sakarya.edu.tr Received: 01.08 2015, Accepted: 20.03.2016

Copyright (C) Journal of Microbiology and Infectious Diseases 2016, All rights reserved
2. Intermittent carriage, affecting about $60 \%$ of the population and involving different strains; and

3. Non-carriage, describing the approximately $20 \%$ of the population that almost never carries $S$. aureus [4].

Health care workers with NCSa constitute a very significant channel for the development of induced staphylococcal infections [1]. Epidemics have been reported due to health-care staff with NCSa [5]. Methicillin-resistant $S$. aureus (MRSa) strains are more often found in health-care workers than in the general population. MRSa strains, are resistant to many other antibiotics. They caused high mortality in cases of severe infection and in those that are difficult to treat, including lengthy hospitalizations and cases requiring extensive use of antibiotics; patients in intensive care, post-operative period, newborn, haemodialysis, and burn units are particularly at risk. NCSa has been found to occur within the first 10 days in approximately $20 \%$ of hospitalized 
patients [6]. The role of medical workers in this colonization is exactly undeniable [7]. Therefore, the determination and isolation of NCSa carriers constitute one of the crucial steps of infection control.

Medical students are often in contact with patients during their internships, and therefore they should also be assumed as health-care workers. Medical education in our country involves six (Turkish programs) or seven years (English programs). Medical students often receive theoretical training in the first three years of their medical education (described in this study as the preclinical phase), and then in the fourth year begin practical training in the teaching and research hospital of their medical faculty. The question arises as to whether beginning practical training could affect the frequency of NCSa in medical students.

We found that there were very few studies addressing this question. In this study, we aimed to investigate and compare the frequency of NCSa in preclinical students (PS) and clinical students (CS).

\section{MATERIALS AND METHODS}

\section{Workplace and design}

This cross-sectional study was conducted at the Sakarya University Faculty of Medicine between January 01, 2014 and May 15, 2014. Before the procedure, consent documentation was obtained from the subjects and a questionnaire was administered using a face-to-face interview technique. The demographic attributes of potential subjects were elicited in the questionnaire as well as various risk factors for carriage. The local ethics committee's approval was obtained for this study (2014/01).

The study population was composed of all the students in the Faculty of Medicine, a total of 205 potential subjects. Of these, 140 were PS, of whom 82 agreed to participate in the study, and 65 were CS, of whom 64 agreed to take part.

\section{Sample collection and procedure}

Samples were taken from one $\mathrm{cm}$ inside the nostrils with three rotations of the swab. The samples were transferred to the laboratory within four hours without using a transport medium; they were then inoculated into the Chapman agar medium, and incubated at $37^{\circ} \mathrm{C}$ for 48 hours. The colonies that grew with a golden-yellow colour were considered as potential $S$. aureus colonies. The identification as an $S$. aureus colony was confirmed by gram staining, by catalase and coagulase tests. Antibiotic sensitivity tests were performed on these colonies. Oxacillin discs were used to determine antibiotic resistance for methicillin resistance. Diameters $<10$ $\mathrm{mm}$ for oxacillin were accepted as indicating methicillin resistance.

Individuals with $S$. aureus in the first swab sample (Sample 1=S1) were informed via e-mail that the result of the first samples were positive and they were asked to give second sample (Sample 2=S2) 7 days after the first sample. The same procedure was performed for the second sample. The following formula was applied to define the carriage.

\section{S1 negative: Non-carriage}

S1 positive but S2 negative for NCSa: Intermittent carriage

Both S1 and S2 positive for NCSa: Persistent carriage

\section{Data analysis}

Data were assessed using statistical software (Epi Info 7.1.3; Centers for Disease Control and Prevention, Atlanta, GA 30333). Categorical variables were compared by Pearson's or Yates corrected ChiSquare tests. Categorical variables were presented as a count and percentage. A p-value $<0.05$ was considered as significant.

\section{RESULTS}

\section{Demographic data}

A total of 205 students (were asked to participate in the study, and $146(71.2 \%)$ of them agreed to do so, 53 subjects were male and 93 were female). Nasal swab samples were taken from a total of 146 students, 82 out of 140 PS and 64 out of 65 CS. The demographic characteristics of persistent carriers and non-carriers are summarized in Table 1.

Table 1. Demographic characteristics of persistent nasal carriers and non-carriers

\begin{tabular}{lccc}
\hline Parameter & $\begin{array}{c}{ }^{*} \mathrm{NCSa}(+) \\
\mathrm{N}=9\end{array}$ & $\begin{array}{c}{ }^{*} \mathrm{NCSa}(-) \\
\mathrm{N}=137\end{array}$ & p-value \\
\hline Sex (Male/Female) & $4 / 5$ & $49 / 88$ & 0.72 \\
Smoking & 1 & 9 & 1.0 \\
Antibiotic usage & 3 & 39 & 0.71 \\
Preclinical/clinical & $2 / 7$ & $80 / 57$ & 0.042 \\
Nasal spray use & 1 & 25 & 1.0 \\
\hline Hospitalization & 1 & 5 & 1.0 \\
\hline
\end{tabular}

${ }^{*}$ Nasal Carriage of Staphylococcus aureus 


\section{Data of NCSa}

In nine subjects, persistent NCSa was detected but intermittent carriage was not. While NCSa frequency within the PS group was $2 / 82(2.4 \%)$, that within the CS group was $7 / 64(10.9 \%)(p=0.042)$. Methicillin resistance was not detected in any subject. The distribution of students according to their years and isolation of $S$. aureus nasal swabs is shown in Figure 1.

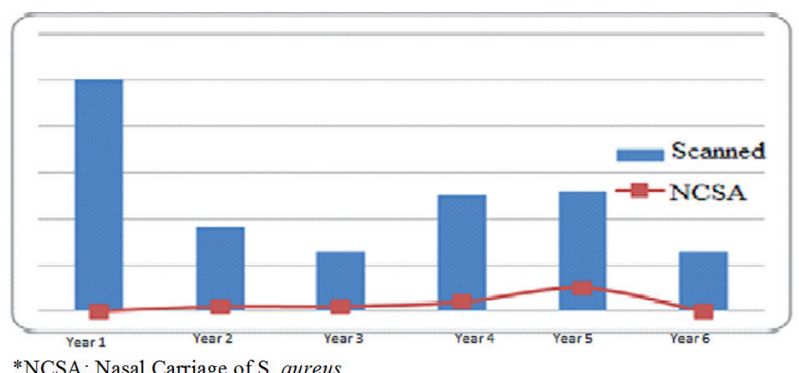

*NCSA: Nasal Carriage of S. aureus

Figure 1. The distribution of students according to $\mathbf{S}$. $\mathbf{a u}$ reus isolation of the year and nasal swab samples

There was no significant difference between the genders in terms of NCSa $(p=0.72)$. Smoking, the use of antibiotics, the use of nasal sprays, and the history of hospitalization had no effect on the rate of NCSa $(p>0.05)$. Aspirin use, diabetes, hypertension, surgical experience in the last six months, skin or soft tissue infection, and immune suppressive drug use were not detected as risk factors in any subject.

\section{DISCUSSION}

In this study, the frequency of NCSa in the CS group $(10.9 \%)$ was found to be about four times higher than that in the PS group (2.4\%). This high frequency among CS subjects might be caused by their interaction with patients and, in particular, by their performing invasive processes during their training, such as inserting a Foley catheter, collecting blood, or collecting nasal and throat swab samples.

Medical students in the fourth and fifth grade are subjected to a test at the end of their internship. Students in these years have more contact with patients than other medical students as they are being trained in order to gain clinical competence, so it might be expected that their NCSa frequency in this study would be higher than that of the other subjects. However, while the NCSa frequency of all students in internship might be expected to be high, in our study the ratio for sixth-grade students was found to be lower than that of fourth- and fifth-grade medical students. We think that medical intern (students at the end of their internship) students in Turkey would be likely to spend a good deal of their time in a desk review because of the Medical Specialization Examination. This might explain a lower NCSa frequency among sixth-grade students as they would have less participation in direct patient care than the fourth- and fifth-grade students. We could not find a relationship between NCSa frequency and factors such as the use of antibiotics, age, gender, and smoking. In a similar study, Treesirichod et al. investigated the patterns of NCSa among medical students before and after their clinical rotation. They found that after the clinical rotation, the prevalence rate of asymptomatic nasal carriage of $S$. aureus increased. Moreover, they were unable to find MRSa colonization in their 128 medical students [8]. We were also unable to determine methicillin resistance in any $S$. aureus strains. This may be attributed to our low number of cases and to the young ages of our medical-student subjects. Another possible reason may be the low frequency of nasal MRSa carriage in the Turkish population [9]. Finally, this situation might be related to the good health status of medical students in general [10].

There are some limitations in this study. We studied a small number of medical students. This situation related with our student's number is limited due to we are a new medical school. If we could study high number student we could report data that are more accurate.

To summarize, NCSa frequency was found to be significantly higher among CS than among PS. Educating medical students about NCSa is important for the health of both the students and the patients. We conclude that medical students are as important from an infection-control perspective as other health care workers such as doctors, nurses, and allied health care workers. Especially risky areas of care (orthopaedics, cardiovascular surgery, neurosurgery, and intensive care, among others) should definitely act in accordance with this information. The CS group must be provided with the training necessary to enable them to comply with infection-control measures.

\section{Acknowledgements}

This project was supported by Sakarya University. We thank to Sakarya University BAP. Project No: 2012-08-00-002. 
This study was presented as a poster at the $6^{\text {th }}$ Eurasian Congress on Infectious Diseases $\left(6^{\text {th }}\right.$ EACID) in Serbia.

Declaration of Conflicting Interests: The authors declare that they have no conflict of interest.

Financial Disclosure: No financial support was received.

\section{REFERENCES}

1. Ammerlaan HSM, Kluytmans JAJW, Wertheim HFL, Nouwen JL, Bonten MJM. Eradication of methicillin-resistant Staphylococcus aureus carriage: a systematic review. Clin Infect Dis 2009; 48: 922-30.

2. Kluytmans J, van Belkum A, Verbrugh $H$. Nasal carriage of Staphylococcus aureus: epidemiology, underlying mechanisms, and associated risks. Clin Microbiol Rev 1997; 10: 505-520.

3. Donker JMW, van der Laan L, Hendriks YJAM, Kluytmans JAJW. Evaluation of Staphylococcus aureus nasal carriage screening before vascular surgery. PLoS One 2012; 7: e38127.

4. Vanden Bergh MF, Yzerman EP, van Belkum A, Boelens HA, Sijmons M, Verbrugh HA. Follow-up of Staphylococcus au- reus nasal carriage after 8 years: redefining the persistent carrier state. J Clin Microbiol 1999; 37: 3133-40.

5. El Helali N, Carbonne A, Naas T, et al. Nosocomial outbreak of staphylococcal scalded skin syndrome in neonates: epidemiological investigation and control. J Hosp Infect 2005; 61: 130-8.

6. Cirkovic I, Stepanovic S, Skov R, Trajkovic J, Grgurevic A, Larsen AR. Carriage and Genetic Diversity of Methicillin-Resistant Staphylococcus aureus among Patients and Healthcare Workers in a Serbian University Hospital. PLoS One 2015; 10: e0127347.

7. Karabay O, Otkun MT, Yavuz MT, Otkun M. Nasal carriage of methicillin-resistant and methicillin-susceptible Staphylococcus aureus in nursing home residents in Bolu, Turkey. West Indian Med J 2006; 55: 183-7.

8. Treesirichod A, Hantagool S, Prommalikit O. Nasal carriage and antimicrobial susceptibility of Staphylococcus aureus among medical students at the HRH Princess Maha Chakri Sirindhorn Medical Center, Thailand: a follow-up study. J Infect Public Health; 7: 205-9.

9. Erdenizmenli M, Yapar N, Senger SS, Ozdemir S, Yuce A. Investigation of colonization with methicillin-resistant and methicillin-susceptible Staphylococcus aureus in an outpatient population in Turkey. Jpn J Infect Dis 2004; 57: 172-5.

10. Trépanier P, Tremblay C, Ruest A. Methicillin-resistant Staphylococcus aureus colonization among medical residents. Can J Infect Dis Med Microbiol 2013; 24: e39-41. 